\title{
Monolayer Characteristics of a Long-Chain N,O-Diacyl Substituted Ethanolamine at the Air/Water Interface
}

\author{
G. Brezesinski, ${ }^{\dagger}$ B. Dobner, ${ }^{\dagger}$ C. Stefaniu, ${ }^{\dagger}$ and D. Vollhardt ${ }^{*}{ }^{+}$ \\ ${ }^{\dagger}$ Max Planck Institute of Colloids and Interfaces, D-14424 Potsdam/Golm, Germany \\ ${ }^{\ddagger}$ Institute of Pharmacy, University Halle-Wittenberg, D-06120 Halle (Saale), Germany
}

ABSTRACT: The $\mathrm{N}$ - and/or O-acylation of amphiphilic ethanolamine attracts particular attention because of its interesting biological, pharmaceutical, and medicinal properties. Tetradecanoic acid-2-[(1oxotetradecyl)amino] ethyl ester (TAOAE) as the selected N,O-diacyl derivative of ethanolamine has been synthesized in order to obtain first information about its main interfacial characteristics, such as the surface pressure-area $(\pi-A)$ isotherms, the morphology of the condensed phase domains, the lattice structure of the condensed phase, and information about the existence of interfacial hydrogen bonds $(-\mathrm{NH} \cdots \mathrm{O}=\mathrm{C}-$ ). The $\pi-A$ isotherms of TAOAE, similar to those of the most usual monolayers of amphiphiles, show a sharp break point $\left(A_{c}\right)$ indicating the first-order phase transition from the fluid (liquid-expanded (LE), gaseous $(\mathrm{G})$ ) to the condensed (liquid-condensed (LC)) phase. On the mesoscopic scale, the dendritic domains homogeneously reflecting suggest an orientation of the alkyl chains perpendicular to the aqueous surface. The grazing incidence X-ray

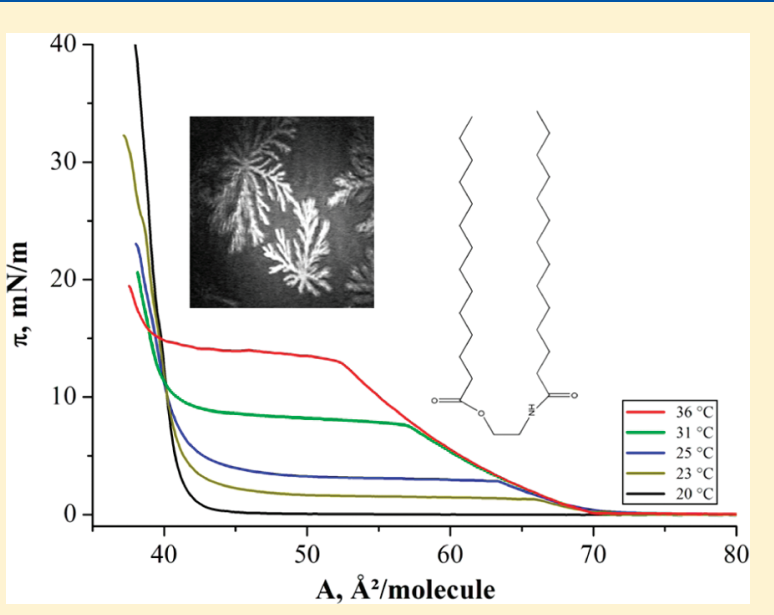
diffraction (GIXD) studies reveal hexagonal packing of the TAOAE molecules oriented perpendicular to the surface in an LS phase. The existence of a hydrogen-bonding network in the monolayer is supported by infrared reflection absorption spectroscopy (IRRAS) experiments.

\section{INTRODUCTION}

Derivatives of amino alcohols and long-chain fatty acids are naturally occurring amphiphiles that are present in a wide variety of animals, plants, and microbes. ${ }^{1,2}$ In nature, there are many myristic acid and hydroxymyristic acid derivatives with ethanolamine that are expected to have lipid A-like activity. ${ }^{3-5}$ These compounds are physiologically attractive materials and drew particular attention to their interesting biological, pharmaceutical, and medicinal properties.

Ethanolamine, having an amino group and a hydroxy group, offers the possibility for $\mathrm{N}$ - and/or $\mathrm{O}$-acylation. ${ }^{6,7}$ A variety of corresponding derivatives have been prepared, and particularly, the synthesis of enzyme-catalyzed N,O-diacyl compounds with various acyl chain lengths attracted attention. ${ }^{5}$ Fatty acid alkanol amides are of great interest for applications requiring relatively stable emulsifiers because amide linkages are chemically very stable. $^{8,9}$ These types of compounds are employed in the manufacture of foods, cosmetics, pharmaceutical products, and a wide variety of specialty chemicals which exploit the surface active properties of these materials. ${ }^{10}$ In particular, several important pharmaceutical products are derivatives of amino alcohols and long-chain fatty acids because of the excellent antimicrobial activities, ${ }^{11-13}$ the favorable biodegradability, and the relatively nonallergenic character. ${ }^{14}$
Despite the numerous papers concerning synthesis, occurrence, and application of derivatives of amino alcohols with longchain fatty acids, there is only little information on the interfacial characteristics of some selected amphiphiles of this type. In fact, we investigated the main monolayer characteristics of various tailored amphiphiles containing an amide group and one or two hydroxyl groups separated by one or more (two or three) methylene groups, ${ }^{15-25}$ but with $\mathrm{N}$-myristoylethanolamine $\left(\mathrm{C}_{13} \mathrm{H}_{27}-\mathrm{CO}-\mathrm{NH}-\mathrm{C}_{2} \mathrm{H}_{4} \mathrm{OH}\right)$ only one $\mathrm{N}$-acylated ethanolamine has been studied. ${ }^{26}$ However, information about monolayer characteristics of long-chain $\mathrm{N}, \mathrm{O}$-diacyl compounds of ethanolamine is completely missing.

The objective of the present work has been to provide a first contribution for filling this gap. We synthesized tetradecanoic acid-2-[(1-oxotetradecyl)amino] ethyl ester $\left(\mathrm{C}_{13} \mathrm{H}_{27}-\mathrm{CO}-\right.$ $\mathrm{NH}-\left(\mathrm{CH}_{2}\right)_{2}-\mathrm{O}-\mathrm{CO}-\mathrm{C}_{13} \mathrm{H}_{27}$, TAOAE, Figure 1) as the selected N,O-diacyl derivative of ethanolamine and determined the main characteristics of its monolayers, such as the surface pressure-area $(\pi-A)$ isotherms, the morphology of the condensed phase domains, the lattice structures of the condensed

\footnotetext{
Received: January 19, 2011

Revised: $\quad$ March 5, 2011

Published: March 30, 2011
} 


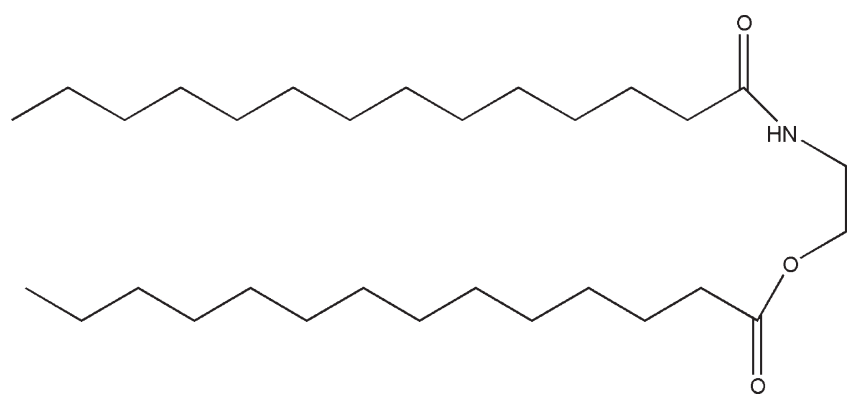

Figure 1. Chemical structure of tetradecanoic acid-2-[(1-oxotetradecyl)amino] ethyl ester (TAOAE).

phase, and information about the existence of hydrogen bonds $(-\mathrm{NH} \cdot \cdots \mathrm{O}=\mathrm{C}-)$ in the monolayer.

\section{EXPERIMENTAL SECTION}

Materials. Synthesis of Tetradecanoic Acid-2-[(1-Oxotetradecyl)amino]ethyl Ester (TAOAE). Amounts of $0.01 \mathrm{~mol}(0.61 \mathrm{~g})$ of 2-aminoethanol and $0.022 \mathrm{~mol}(2.2 \mathrm{~g})$ of TEA were dissolved in $50 \mathrm{~mL}$ of chloroform. The mixture was cooled down to $0{ }^{\circ} \mathrm{C}$, and $0.022 \mathrm{~mol}(5.43 \mathrm{~g})$ of tetradecanoic acid chloride dissolved in $20 \mathrm{~mL}$ of $\mathrm{CHCl}_{3}$ was added within 20 min under stirring. The mixture was then stirred for a further $30 \mathrm{~min}$ at $0{ }^{\circ} \mathrm{C}$ and subsequently for $2 \mathrm{~h}$ at room temperature. The mixture was then poured into saturated ammonium chloride solution, and the organic layer was separated, dried over sodium sulfate, and evaporated. The residue was purified by column chromatography. Yield: $3.75 \mathrm{~g}(77.8 \%)$ of white crystals; mp, $82-83{ }^{\circ} \mathrm{C}$. ESIMS: $504.37\left(100 \%,[\mathrm{M}+\mathrm{Na}]^{+}\right)$. Elemental analysis: Calcd: C, 74.80; H, 12.34; N, 2.90. Found: C, 74.81; H, 12.23; N, 2.77. ${ }^{1} \mathrm{H}$ $\operatorname{NMR}\left(400 \mathrm{MHz}, \mathrm{CDCl}_{3}\right): \delta=0.85\left(\mathrm{t}, 6 \mathrm{H}, 2 \times\left[\mathrm{CH}_{3}-\right]\right), 1.2-1.35(\mathrm{~m}$, $40 \mathrm{H}$, [chain]), $1.5-1.65\left(\mathrm{~m}, 4 \mathrm{H},\left[-\mathrm{NH}-\mathrm{CO}-\mathrm{CH}_{2} \mathrm{CH}_{2}-\right][-\mathrm{O}-\right.$ $\left.\left.\mathrm{CO}-\mathrm{CH}_{2} \mathrm{CH}_{2}-\right]\right), 2.1-2.2\left(\mathrm{t}, 2 \mathrm{H},\left[-\mathrm{NH}-\mathrm{CO}-\mathrm{CH}_{2}-\right]\right), 1.25-2.35$ $\left(\mathrm{t}, 2 \mathrm{H},\left[-\mathrm{O}-\mathrm{CO}-\mathrm{CH}_{2}-\right]\right), 3.45-3.54$ (q, $2 \mathrm{H}, \quad\left[-\mathrm{CH}_{2}-\mathrm{O}-\right.$ $\mathrm{CO}]$ ), $4.1-4.19\left(\mathrm{~m}, 2 \mathrm{H},\left[-\mathrm{CH}_{2}-\mathrm{NH}-\mathrm{CO}-\right]\right), 5.65-5.73(\mathrm{~m}, 1 \mathrm{H}$, $[-\mathrm{NH}-]) \mathrm{ppm}$.

The used spreading solvent was chloroform (p.a. grade, Baker, Holland). Ultrapure water produced by "Purelab Plus" was used as subphase.

Surface Pressure Measurements and Brewster Angle Microscopy. An experimental setup consisting of a self-made computer-interfaced film balance coupled with a Brewster angle microscope (BAM1+, NFT, Göttingen, Germany) was used to measure the equilibrium surface pressure $(\pi-A)$ isotherms at a compression rate of $\leq 10 \AA^{2} /$ (molecule $\cdot \min ) .{ }^{32}$ According to the Wilhelmy method, the surface pressure was measured with a roughened glass plate with an accuracy of $\pm 0.1 \mathrm{mN} \mathrm{m}^{-1}$, and the area per molecule with $\pm 0.5 \AA^{2}$.

The lateral resolution of the BAM1+ was approximately $4 \mu \mathrm{m}$. Simple imaging processing software was used to improve the contrast. Detailed information about the BAM method is given elsewhere (see refs 27-29).

Infrared Reflection Absorption Spectroscopy Measurements ${ }^{30-33}$. The measured signal in infrared reflection absorption spectroscopy (IRRAS) represents the ratio of the reflected light from two liquid surfaces: $\mathrm{RA}=-\log [($ sample reflectivity $) /($ reference reflectivity $)]=-\log \left(R / R_{0}\right)$ in reflectance-absorbance (RA) units. The principle of the method and its application to Langmuir films at the air-water interfaces are described in ref 30 .

The measurements were performed using an IFS 66 FTIR spectrometer equipped with a liquid nitrogen cooled mercury cadmium telluride detector attached to an external air/water reflection unit (XA-511,

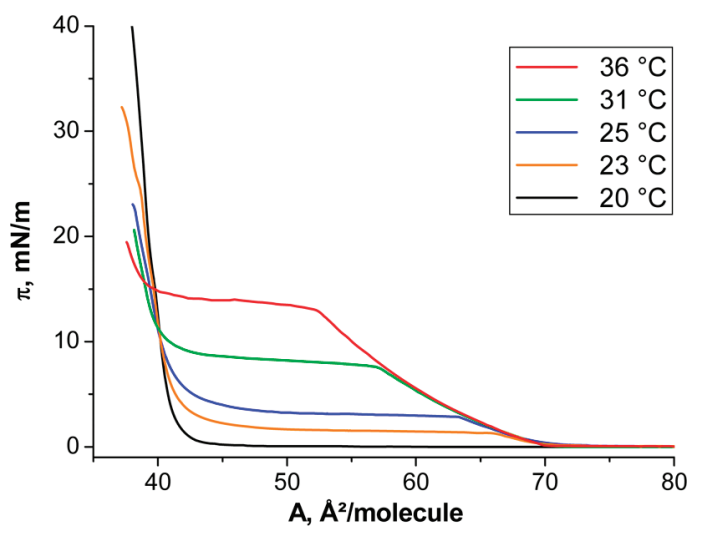

Figure 2. $\pi-A$ isotherms of tetradecanoic acid-2-[(1-oxotetradecyl)amino] ethyl ester (TAOAE) monolayers on water measured in the temperature range between 20 and $36^{\circ} \mathrm{C}$.

Bruker). A small reference trough and the larger sample trough are alternatively moved into the IR beam path by a shuttling mechanism. The resolution and scanner speed in all experiments were $8 \mathrm{~cm}^{-1}$ and $20 \mathrm{kHz}$. The incident IR beam is polarized with a KRS-5 wire grid polarizer. Spectra are coadded over 200 scans for s-polarized light and over 400 scans for p-polarized light. The two different light polarizations provide information on molecular orientation with respect to the surface plane of the monolayer. A change in the intensity ratio of p-polarized to $\mathrm{s}$-polarized light $(\mathrm{p} / \mathrm{s}$ ratio) for a vibrational band indicates a change in the average orientation of the vibration and thus of the molecule.

$X$-ray Diffraction Measurements ${ }^{34-37}$. The grazing incidence X-ray diffraction (GIXD) experiments were performed using the liquid surface diffractometer on the undulator beamline BW1 at HASYLAB, DESY, Hamburg, Germany. In a thermostatted Langmuir film balance placed in a hermetically closed container filled with helium, a monochromatic synchrotron X-ray beam $(\lambda=1.304 \AA)$ is adjusted to strike the helium/ water interface at a grazing incidence angle $\alpha_{i}=0.85 \alpha_{c}\left(\alpha_{c}=0.13^{\circ}\right.$ is the critical angle for total reflection) illuminating approximately $2 \times 50 \mathrm{~mm}^{2}$ monolayer surface. A MYTHEN detector system (PSI, Villigen, Switzerland) measures the diffracted signal and is rotated to scan the in-plane $Q_{x y}$ component values of the scattering vector. A Soller collimator in front of the MYTHEN restricted the in-plane divergence of the diffracted beam to $0.09^{\circ}$. The vertical strips of the MYTHEN measure the out-of-plane $Q_{z}$ component of the scattering vector between 0.0 and $0.75 \AA^{-1}$. The diffraction data consist of Bragg peaks at diagnostic $Q_{x y}$ values. These peaks are calculated by summing the diffracted intensity at each in-plane $Q_{x y}$ value over a defined vertical angle or $q_{z}$-window. The in-plane lattice repeat distances $d$ of the ordered structures in the monolayer are calculated from the Bragg peak positions: $d=2 \pi / Q_{x y}$. To access the extent of the crystalline order in the monolayer, the in-plane coherence length $L_{x y}$, is approximated from the full width at half-maximum (fwhm) of the Bragg peaks using $L_{x y} \sim$ $0.9(2 \pi) /$ fwhm $\left(Q_{x y}\right)$. The diffracted intensity normal to the interface is integrated over the $Q_{x y}$ window containing the diffraction peak to calculate the corresponding Bragg rod. The thickness of the monolayer is estimated from the fwhm of the Bragg rod using $0.9(2 \pi) / f w h m\left(Q_{z}\right)$.

\section{RESULTS AND DISCUSSION}

The experimental $\pi-A$ isotherms of the TAOAE monolayers on pure water, measured at different temperatures in the range between 15 and $36^{\circ} \mathrm{C}$, are shown in Figure 2 .

The features of the $\pi-A$ isotherms of TAOAE correspond to those of most usual amphiphiles. ${ }^{38}$ The isotherms show a sharp inflection point $\left(A_{\mathrm{c}}\right)$ indicating the starting point of the 


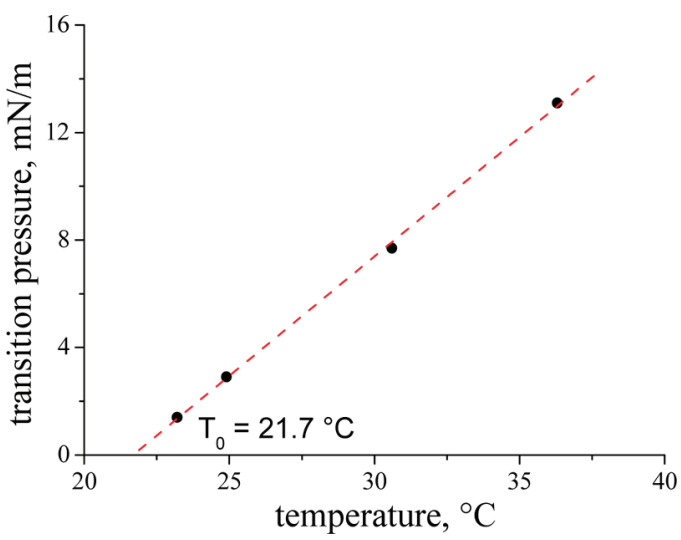

Figure 3. Temperature dependence of the main phase transition pressure $\pi_{\mathrm{t}}$ of TAOAE monolayers spread on water.

first-order phase transition from the fluid (liquid-expanded (LE), gaseous $(\mathrm{G})$ ) to the condensed (liquid-condensed (LC)) phase. At all temperatures $\geq 21.7^{\circ} \mathrm{C}$, an extended horizontal plateau region is observed, whereas in many amphiphilic monolayers the two-phase transition region is increasingly inclined with increasing temperature. However, the extension of the phase transition region of the TAOAE monolayers decreases in the usual way as the temperature increases. At $T<21.7^{\circ} \mathrm{C}$, the condensed phase is already in coexistence with the fluid phase at zero pressure.

The changed slope of the isotherms in the condensed part at higher temperatures indicates that the solubility of TAOAE increases with increasing temperature. A small amount of material is lost during compression. The estimation of the area per molecule in the condensed state at the end of the first-order phase transition is therefore connected with certain error bars.

The temperature dependence of the phase transition pressure $\left(\pi_{\mathrm{c}}\right)$ (the kink point in the $\pi-A$ isotherm at the onset of the phase transition) provides information on the phase behavior and the thermodynamic characteristics of the transition between the fluid and condensed phase.

Figure 3 shows the $\pi_{\mathrm{t}}-T$ relationship of TAOAE monolayers spread on water fitted by linear regression. The slope $d \pi_{c} / d T$ of this line amounts to $0.888 \mathrm{mN} /(\mathrm{m} \cdot \mathrm{K})$.

The two-dimensional (2D) Clapeyron equation representing a one-component approximation can be used for calculating the enthalpy change $\Delta H$ of the phase transition

$$
\Delta H=\left(A_{\mathrm{c}}-A_{\mathrm{e}}\right) T \frac{\mathrm{d} \pi_{\mathrm{t}}}{\mathrm{d} T}
$$

where $A_{\mathrm{e}}$ is the molecular area at the onset of the phase transition at the surface pressure $\pi_{\mathrm{t}}$ and $A_{\mathrm{c}}$ is the area of the condensed phase at this pressure. The temperature dependence of the entropy change $\Delta S=\Delta H / T$ for the phase transition is presented in Figure 4. Negative $\Delta H$ and $\Delta S$ values are obtained according to the exothermic nature of the main phase transition at compression of amphiphilic monolayers and an increase in the ordering of the system. The absolute $\Delta S$ and $\Delta H$ values increase as the temperature decreases indicating that the ordering of the condensed phase increases as the temperature decreases. The critical temperature of $48.6^{\circ} \mathrm{C}$, above which the monolayer cannot exists in the condensed state, results from the extrapolation to $\Delta S=0$.

Figures 2 and 3 show that the $\mathrm{C}_{13} \mathrm{H}_{27}$ alkyl chain of the N,Odiacyl derivative of ethanolamine is sufficiently long to have the

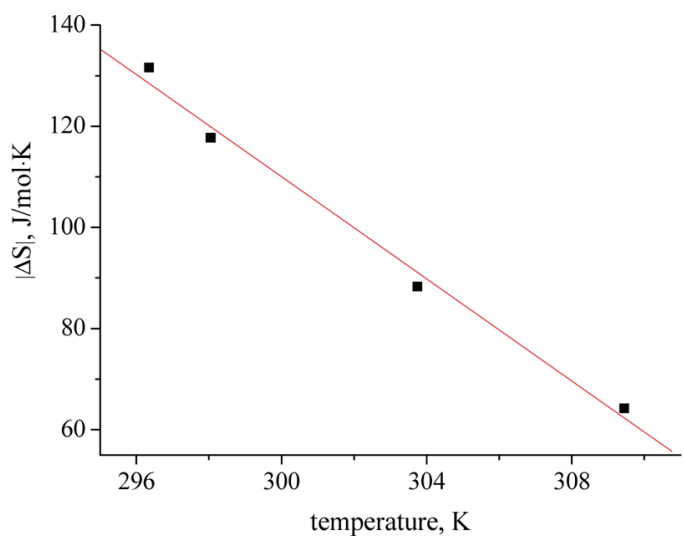

Figure 4. Temperature dependence of the entropy change for the main phase transition of TAOAE monolayers.

fluid/condensed phase coexistence region in the accessible temperature range above $22^{\circ} \mathrm{C}$ and, thus, to provide reasonable conditions for studying the domain morphology using Brewster angle microscopy.

Figure 5 shows different growth steps of typical TAOAE domains which are formed after the main phase transition point within the fluid/condensed transition region (see Figure 1) at a slow compression rate of $1 \AA^{2} /($ molecule $\cdot \min )$ at $23{ }^{\circ} \mathrm{C}$. The bright condensed phase domains are surrounded by the fluid phase of lower density and thus lower reflectivity. At the beginning of the growth process, several main axes grow from a small round center (Figure 5a), but very soon further main axes and numerous side arms in both directions develop (Figure $5 b$ ). The dendritic domain shape is obviously less crystalline and largely independent of the temperature. The less crystalline nature of the domain shape and the absence of an inner texture of the domains become apparent in the further stages of the domain growth (Figure 5, parts $\mathrm{c}$ and $\mathrm{d}$ ). It is interesting to note that the bright TAOAE domains are homogeneously reflecting. This suggests an orientation of the alkyl chains perpendicular to the aqueous surface. The stability of the dendritic domains is remarkably high so that a rearrangement to a compact equilibrium shape could not be observed. Despite the specific character of the TAOAE domains, this behavior is in agreement with experimental results of other amphiphiles containing an acid amide group. ${ }^{15-18,21,22}$

GIXD studies provide information about the characteristic features of the lattice structure of condensed monolayer phases of long-chain N,O-diacyl substituted ethanolamine on the angstrom scale. Figure 6 demonstrates the results for a TAOAE monolayer measured at $\pi=10 \mathrm{mN} / \mathrm{m}$ and a temperature of $5{ }^{\circ} \mathrm{C}$. The diffracted intensity is plotted as contour lines of equal intensity versus the in-plane component $Q_{x y}$ and the out-ofplane component $Q_{z}$ of the scattering vector (Figure 6, bottom left), as a function of the in-plane scattering vector component $Q_{x y}$ (Bragg peak, Figure 6 top), and as a function of the outof-plane scattering vector component $Q_{z}$ (Bragg rod, Figure 4 bottom right).

Number and position of the reflexes in the contour plot allow first qualitative conclusions. At all accessible lateral pressures, only one diffraction peak was observed, indicating hexagonal packing of the TAOAE molecules oriented perpendicular to the surface in an LS phase. Integration over the whole $Q_{z}$-window of the detector and fitting a Lorentzian function to the experimental 


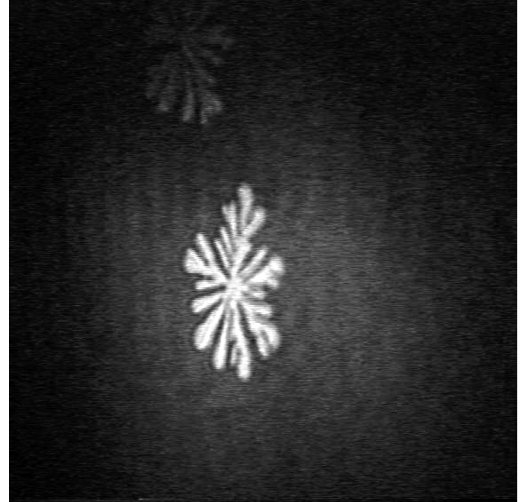

(a)

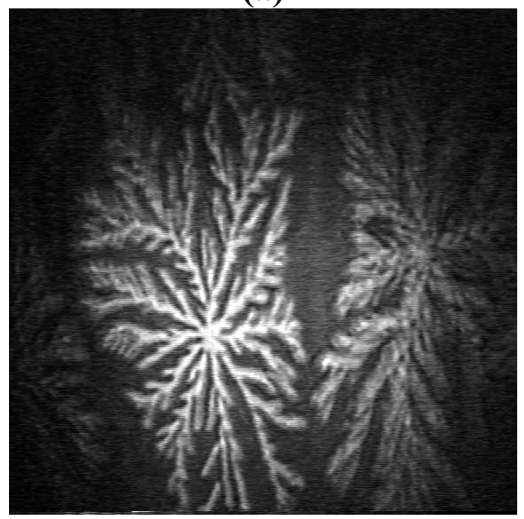

(c)

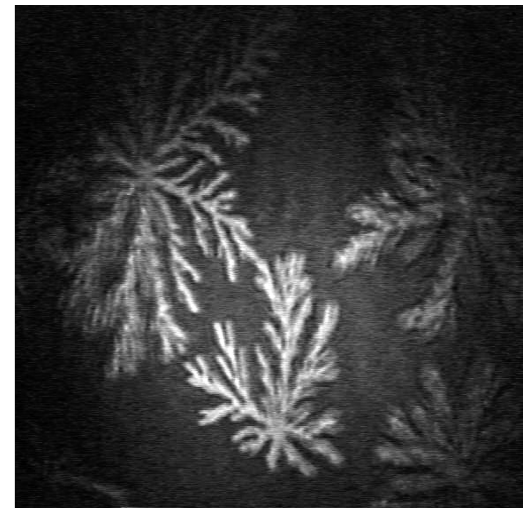

(b)

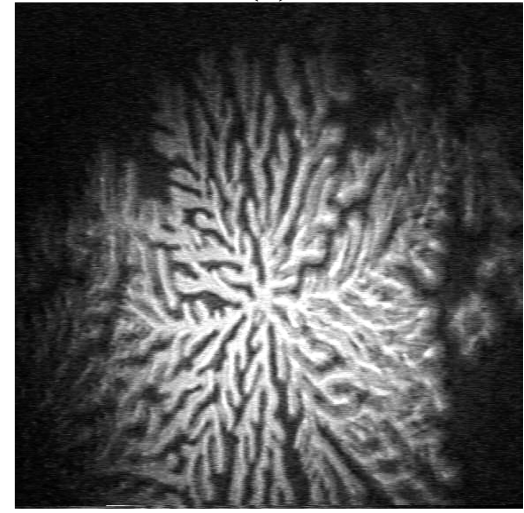

(d)

Figure 5. Growth steps of typical TAOAE domains formed after the main phase transition point within the fluid/condensed transition region. Compression rate: $1 \AA^{2} /($ molecule $\cdot \min ), T=23^{\circ} \mathrm{C}$.

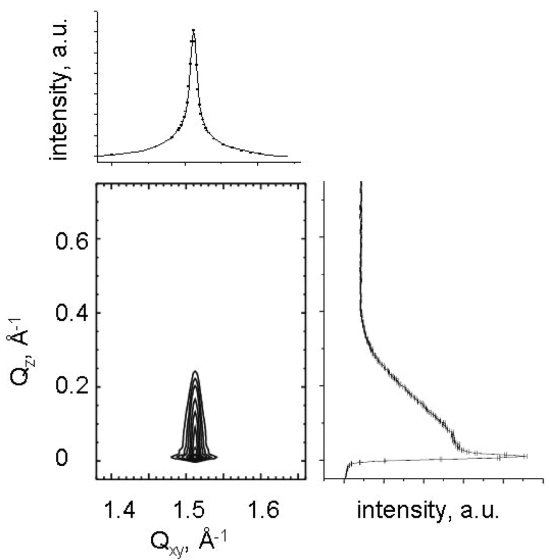

Figure 6. Grazing incidence X-ray diffraction data for a TAOAE monolayer spread on water at $5^{\circ} \mathrm{C}$ and compressed to $10 \mathrm{mN} \mathrm{m}^{-1}$. The diffracted intensities, corrected for polarization, effective area, and Lorentz factor, are plotted as contour lines of equal intensity vs the inplane component $Q_{x y}$ and the out-of-plane component $Q_{z}$ of the scattering vector (bottom left). The Bragg peak (top) and the Bragg rod (bottom right), which is superimposed by the sharp Yoneda-Vineyard peak, are also shown.

data show that this peak is located at a $Q_{x y}$-value of $1.512 \AA^{-1}$. For selected lateral pressures of 4 and $10 \mathrm{mN} / \mathrm{m}$ at $5{ }^{\circ} \mathrm{C}$, the Bragg peak positions, their full widths at half-maximum, fwhm$\left(Q_{x y}\right)$, and the lattice parameters are listed in Table 1 . As the data of Table 1 show, the position of the Bragg peak shifts only slightly to larger $Q_{x y}$ values as the surface pressure increases. Therefore, the area of the unit cell, $A_{x y}$, decreases very slightly from $20.0 \AA^{2}$ at $4 \mathrm{mN} / \mathrm{m}$ to $19.9 \AA^{2}$ at $10 \mathrm{mN} / \mathrm{m}$ showing that the condensed phase is almost incompressible. These values of the crosssectional alkyl chain area indicate a certain mobility of the alkyl chains in a rotator phase. Assuming that the position of the Bragg peak depends linearly on the lateral pressure, the compression modulus $K$ can be directly determined by $K=1 / 2 Q_{x y}(\Delta \pi /$ $\left.\Delta Q_{x y}\right)^{39-41}$ and amounts to $2.3 \mathrm{~N} / \mathrm{m}$. This value is similar to that observed for hexadecanol in the LS phase at $32{ }^{\circ} \mathrm{C}$. Since the decrease of the alkyl chain length by two methylene groups is equivalent to a temperature increase, the similarity of the $K$ values indicates that, in both cases, the compressibility in the LS phase is determined by the alkyl chains which occupy a larger or similar area as the head groups.

The single Bragg peak describes a hexagonal packing of the alkyl chains of the TAOAE molecules in the monolayers (LS phase). Assuming that the monolayer consists of $2 \mathrm{D}$ crystallites that are perfect and have a finite size $L_{x y}$, this size can be estimated by $L_{x y} \sim(0.92 \pi) /$ fwhm $\left(Q_{x y}\right)$. Assuming an exponential decay of the correlation, as found in liquid crystals, the correlation length $\xi=2 /$ fwhm $\left(Q_{x y}\right)$ can be calculated. The measured fwhm $\left(Q_{x y}\right)$ corrected by the instrumental resolution of the Soller collimator leads to a size of the $2 \mathrm{D}$ crystallites of $L_{x y} \approx$ $378 \AA(\xi \sim 133 \AA)$ at $4 \mathrm{mN} / \mathrm{m}$ and of $L_{x y} \approx 333 \AA(\xi \sim 118 \AA)$ at $10 \mathrm{mN} / \mathrm{m}$ in the observed LS phase. The decrease of the correlation length shows that the compression of the selfassembled monolayer disturbs the perfect packing by creating more defects. These $L_{x y}$ values are smaller compared with those 
Table 1. GIXD Results of TAOAE Monolayers

\begin{tabular}{|c|c|c|c|c|c|c|c|c|c|}
\hline \multirow[b]{2}{*}{$T\left({ }^{\circ} \mathrm{C}\right)$} & \multirow[b]{2}{*}{$\pi(\mathrm{mN} / \mathrm{m})$} & \multicolumn{2}{|c|}{ peak position } & \multicolumn{2}{|c|}{ full width at half-maximum } & \multicolumn{2}{|c|}{ cell parameters } & \multirow{2}{*}{$\begin{array}{c}\text { cross section/unit cell area } \\
A_{x y}=A_{0}\left(\AA^{2}\right)\end{array}$} & \multirow[b]{2}{*}{ tilt (deg) } \\
\hline & & $Q_{x y}\left(\AA^{-1}\right)$ & $Q_{z}\left(\AA^{-1}\right)$ & $\Delta Q_{x y}\left(\AA^{-1}\right)$ & $\Delta Q_{z}\left(\AA^{-1}\right)$ & $a=b=c(\AA)$ & $\alpha=\beta=\gamma(\operatorname{deg})$ & & \\
\hline 5 & 4 & 1.510 & 0 & 0.017 & 0.27 & 4.805 & 120 & 20.0 & 0 \\
\hline 5 & 10 & 1.512 & 0 & 0.019 & 0.28 & 4.798 & 120 & 19.9 & 0 \\
\hline
\end{tabular}

of typical well-packed amphiphilic monolayers consisting of alkyl chain(s) and a polar headgroup, such as fatty acids, but larger than that of the monolayer of the triterpenoid oleanolic acid with a multicyclic planar structure. ${ }^{42,43}$

For characterizing the $2 \mathrm{D}$ packing features of the monolayer the thickness, $L_{z}$, of the TAOAE layer is of further interest. The thickness of the diffracting layer can be estimated from the full width at half-maximum (fwhm) of the Bragg rod (Figure 6, bottom right) using $L_{z} \sim 0.9(2 \pi) / \mathrm{fwhm}\left(Q_{z}\right)$. The average value of the fwhm $\left(Q_{z}\right)$ of the TAOAE monolayer amounts to $0.275 \AA^{-1}$ yielding a thickness of $20.2 \AA$ for the part contributing to the observed diffraction signal of the hydrophobic chains.

The 2D lattice structure of the selected long-chain N,O-diacyl substituted ethanolamine TAOAE reveals obvious differences to the acid amide amphiphiles having only one alkyl chain. The single Bragg peak indicates hexagonal packing of the alkyl chains of the TAOAE molecules in the monolayers (LS phase). The $\pi-A$ isotherms (Figure 2) show that no second phase transition within the condensed phase region exists. On the other hand, acid amide amphiphiles having only one alkyl chain form an oblique lattice structure after the main phase transition point. At low temperatures they can have a second phase transition of first order accompanied by a jumplike change of the tilt angle and the cross-sectional area.

IRRAS experiments have been performed to obtain additional information. It is well-known that $\mathrm{H}$-bonds shift the bands of the stretching vibrations $v(\mathrm{NH})$ and $v(\mathrm{C}=\mathrm{O})$ of amides to lower wavenumbers and that of the deformation peak $\delta(\mathrm{NH})$ in the opposite direction. For secondary amides, the $v(\mathrm{NH})$ peak has different locations for trans- and cis-conformations of the amide group. The trans-isomer, whose $\mathrm{N}-\mathrm{H}$ and $\mathrm{C}=\mathrm{O}$ bonds have opposite orientation, exposes the free $\mathrm{NH}$ stretching peak in dilute solution at $3460-3400 \mathrm{~cm}^{-1}$. The cis-isomer with $\mathrm{N}-\mathrm{H}$ and $\mathrm{C}=\mathrm{O}$ bonds pointing in the same direction exhibits the free $\mathrm{NH}$ stretching peak in the range of $3440-3300 \mathrm{~cm}^{-1}$.

For the comparison with the interfacial results and to facilitate the analysis of IRRA spectra the IR transmission spectrum of TAOAE in $\mathrm{CCl}_{4}$ was also measured (Perkin-Elmer spectrum BX FTIR system, 100 scans, $1 \mathrm{~cm}^{-1}$ resolution, $\mathrm{KBr}$ cell with 0.215 $\mathrm{mm}$ path length). The $\mathrm{NH}$ stretching peak is located at $3462 \mathrm{~cm}^{-1}$ clearly indicating that the $\mathrm{N}-\mathrm{H}$ and $\mathrm{C}=\mathrm{O}$ bonds point in different directions. The $\mathrm{CH}$ stretching vibration region $\left(2840-2975 \mathrm{~cm}^{-1}\right)$ is characterized by two prominent bands (symmetric and asymmetric $\mathrm{CH}_{2}$ stretching vibrations). The symmetric and asymmetric $\mathrm{CH}_{2}$ stretching bands are located at 2855 and $2927 \mathrm{~cm}^{-1}$, respectively, showing that the chains are disordered in solution. Saturated aliphatic esters absorb at $1750-1725 \mathrm{~cm}^{-1}$. In the present case, the ester band is located at $1742 \mathrm{~cm}^{-1}$. The amide band due to the $\mathrm{C}=\mathrm{O}$ stretching vibration (amide I band) of secondary amides in dilute solution is usually found at $1700-1665 \mathrm{~cm}^{-1}$. In the present case, this band is located at $1686 \mathrm{~cm}^{-1}$. The amide II band (due to $\mathrm{N}-\mathrm{H}$ deformation, which plays the major role, and $\mathrm{C}-\mathrm{N}$ stretching

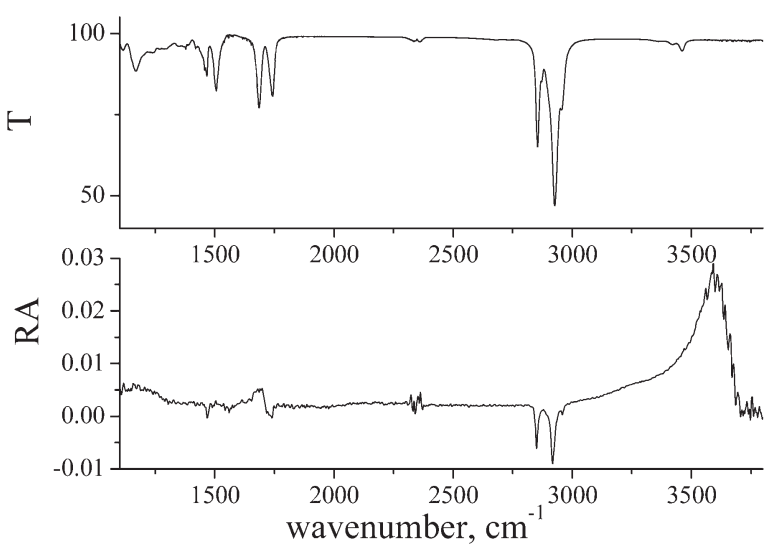

Figure 7. Transmission IR spectrum (top) and IRRA spectrum (bottom) of TAOAE in $\mathrm{CCl}_{4}$ and as a monolayer on water at $10 \mathrm{mN} / \mathrm{m}$, respectively. The IRRA spectrum is measured at $5{ }^{\circ} \mathrm{C}$ with p-polarized light at an angle of incidence of $45^{\circ}$.

vibrations) of secondary amides in dilute solution can be expected at $1550-1510 \mathrm{~cm}^{-1}$ and is found at $1506 \mathrm{~cm}^{-1}$ in our case. Additionally, the $\mathrm{C}-\mathrm{O}-\mathrm{C}$ asymmetric stretching vibration of the aliphatic ester can be seen at $1169 \mathrm{~cm}^{-1}$, and the much weaker symmetric stretching vibration is located at $1115 \mathrm{~cm}^{-1}$.

Figure 7 shows IR transmission spectrum of TAOAE dissolved in $\mathrm{CCl}_{4}$ together with the IRRA spectrum of the TAOAE monolayer at $10 \mathrm{mN} / \mathrm{m}$ in the condensed LS phase. The most noticeable feature in the IRRA spectrum is the band at $3580 \mathrm{~cm}^{-1}$ that arises from the $\mathrm{OH}$ stretch of water and is a characteristic feature of IRRA spectra. The water $\mathrm{OH}$ stretching vibration present in the reference signal $\left(R_{0}\right)$ is reduced in the reflectivity signal from the monolayer-covered surface $(R)$ because the lipid layer replaces a water layer and masks partially the $\mathrm{OH}$ stretching vibration. The result is a strong positive band at $3580 \mathrm{~cm}^{-1}$ that is related to the monolayer's effective thickness.

The CH stretching vibration region $\left(2840-2975 \mathrm{~cm}^{-1}\right)$ is presented in Figure 8 and is characterized by two prominent bands (symmetric and asymmetric $\mathrm{CH}_{2}$ stretching) and one much weaker band (asymmetric $\mathrm{CH}_{3}$ stretching). Compared to the transmission spectrum in $\mathrm{CCl} 4$, the bands are clearly shifted to lower wavenumbers. The positions of the $\mathrm{CH}_{2}$ stretching bands at 2850 and $2918 \mathrm{~cm}^{-1}$ for the symmetric and asymmetric stretching vibrations, respectively, indicate a well-packed condensed monolayer with all-trans conformation of the alkyl chains. The $\mathrm{CH}_{3}$ asymmetric stretching is located at $2958 \mathrm{~cm}^{-1}$. The asymmetric $\mathrm{CH}_{2}$ deformation band appears as a single peak at $1468 \mathrm{~cm}^{-1}$ (Figure 9). This is in accordance with the observed hexagonal packing in the LS phase, since only an orthorhombic unit cell would lead to a splitting of this band.

The position of the $v(\mathrm{C}=\mathrm{O})$ band (amide $\mathrm{I}$ ) of $\mathrm{R}_{1}-\mathrm{NH}-$ $\mathrm{CO}-\mathrm{R}_{2}$ amides is very sensitive to molecular association. The 


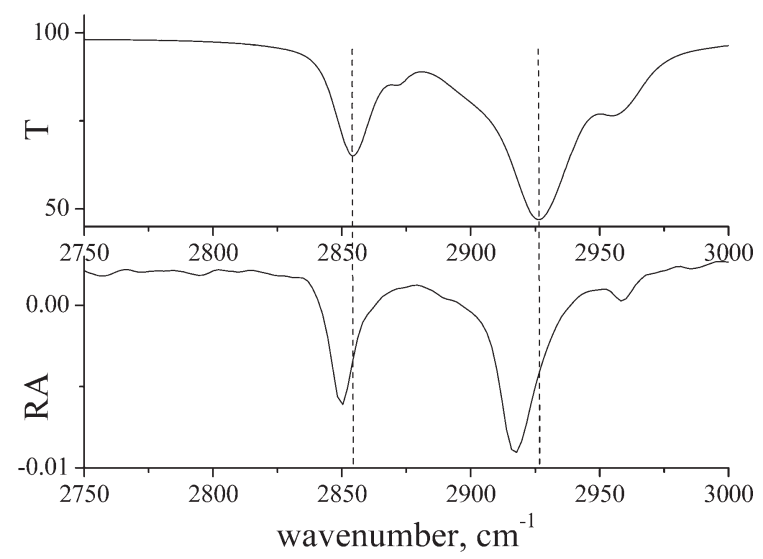

Figure 8. $\mathrm{CH}$ stretching vibration region of transmission $(\mathrm{T})$ and IRRA (RA) spectra of TAOAE in $\mathrm{CCl}_{4}$ or as a monolayer at $10 \mathrm{mN} / \mathrm{m}$ measured at $5{ }^{\circ} \mathrm{C}$ with p-polarized light at an angle of incidence of $45^{\circ}$.

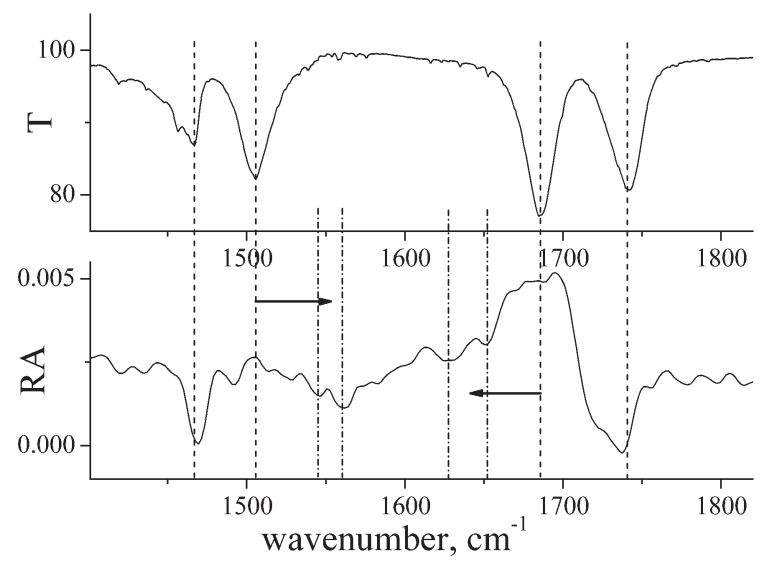

Figure 9. Selected region of transmission (T) and IRRA (RA) spectra of TAOAE in $\mathrm{CCl}_{4}$ or as a monolayer at $10 \mathrm{mN} / \mathrm{m}$ measured at $5{ }^{\circ} \mathrm{C}$ with p-polarized light at an angle of incidence of $45^{\circ}$.

formation of hydrogen bonds $(-\mathrm{NH} \cdots \mathrm{O}=\mathrm{C}-$ ) shifts the amide I band to lower wavenumbers and the amide II band to higher wavenumbers. The existence of a hydrogen bonds in the monolayer is supported by the positions of the amide I (1627 and $1652 \mathrm{~cm}^{-1}$ ) and amide II (1542 and $1561 \mathrm{~cm}^{-1}$ ) bands (Figure 9). The amide I bands are shifted into the direction of lower wavenumbers compared with the spectrum measured in $\mathrm{CCl}_{4}$. The bands at 1627 and $1561 \mathrm{~cm}^{-1}$ show the existence of strongly hydrogen-bonded $\mathrm{C}=\mathrm{O}$ groups. The spectrum is quite noisy, and the intensity of the amide bands is very weak. Nevertheless, a splitting of the bands into two main contributions can be detected. Obviously, the strength of hydrogen bonds seems to be not the same in the whole sample leading to a splitting of the bands.

The ester band observed at $1742 \mathrm{~cm}^{-1}$ in $\mathrm{CCl}_{4}$ solution splits into two components in the monolayer (the band is very asymmetric supporting the assumption of splitting as in the case of the amide bands). One component at $1738-1740 \mathrm{~cm}^{-1}$ shows the existence of free $\mathrm{C}=\mathrm{O}$ stretching vibrations, whereas the other component at $1723-1725 \mathrm{~cm}^{-1}$ can be taken as a proof for the presence of hydrogen bonding.

\section{CONCLUSIONS}

Ethanolamine, having an amino group and a hydroxy group, offers the possibility for $\mathrm{N}$ - and/or O-acylation. Therefore, occurrence and application of the derivatives of amino alcohols with long-chain fatty acids attracted far-reaching interest. However, so far information about the monolayer characteristics of long-chain N,O-diacyl compounds of ethanolamine has been completely missing.

For providing a first contribution to fill this gap we synthesized tetradecanoic acid-2-[(1-oxotetradecyl)amino] ethyl ester (TAOAE) as the selected $\mathrm{N}, \mathrm{O}$-diacyl derivative of ethanolamine and determined the main characteristics of its monolayers, such as the surface pressure-area $(\pi-A)$ isotherms, the morphology of the condensed phase domains, the lattice structures of the condensed phase, and information about the existence of hydrogen bonds $(-\mathrm{NH} \cdots \mathrm{O}=\mathrm{C}-)$ in the monolayer.

The $\pi-A$ isotherms of TAOAE correspond to those of usual monolayers of amphiphiles having a sharp inflection point $\left(A_{\mathrm{c}}\right)$ which indicates the first-order phase transition point from the fluid (LE, G) to the condensed (LC) phase. Enthalpy and entropy changes during the main phase transition have been determined using a 2D Clapeyron equation.

The dendritic domains are homogeneously reflecting, obviously less crystalline, and largely independent of the temperature, suggesting an orientation of the alkyl chains perpendicular to the aqueous surface.

The characteristic features of the lattice structure were obtained by GIXD studies. Only one diffraction peak was observed indicating hexagonal packing of the alkyl chains of the TAOAE molecules oriented perpendicular to the surface in an LS phase. The values of the cross-sectional area reveal a certain mobility of the alkyl chains in a rotator phase. The decreasing size of the $2 \mathrm{D}$ crystallites estimated on the basis of the fwhm shows that the compression of the self-assembled monolayer disturbs the perfect packing by creating more defects.

IRRAS experiments provided additional information about the existence of hydrogen bonds $(-\mathrm{NH} \cdots \mathrm{O}=\mathrm{C}-$ ) in the monolayer. The existence of a hydrogen-bonding network in the monolayer is supported by the positions of the amide I (1627 and $1652 \mathrm{~cm}^{-1}$ ) and amide II $\left(1542\right.$ and $\left.1561 \mathrm{~cm}^{-1}\right)$ bands compared with those obtained in $\mathrm{CCl}_{4}$ solution. The positions of the $\mathrm{CH}_{2}$ stretching bands at 2850 and $2918 \mathrm{~cm}^{-1}$ for the symmetric and asymmetric stretching vibrations, respectively, clearly indicate a well-packed condensed monolayer with alltrans conformation of the alkyl chains. The asymmetric $\mathrm{CH}_{2}$ deformation band as a single peak at $1468 \mathrm{~cm}^{-1}$ is in accordance with the observed hexagonal packing in the LS phase, since an orthorhombic unit cell would lead to a splitting of this band.

\section{AUTHOR INFORMATION}

\section{Corresponding Author}

*E-mail: dieter.vollhardt@mpikg.mpg.de.

\section{ACKNOWLEDGMENT}

We thank HASYLAB at DESY, Hamburg, Germany, for beam time and excellent support. We thank Professors H. H. Rüttinger, M. Lunow, and H. Rudolf, University Halle-Wittenberg, Institute of Pharmacy, for the IR measurements in $\mathrm{CCl}_{4}$ solution. This work was supported by the Max Planck Society. 


\section{REFERENCES}

(1) Schmid, H. H. O.; Schmid, P. C.; Natarajan, V. Prog. Lipid Res. 1990, 29, 1.

(2) Hansen, H. S.; Moesgaard, B.; Hansen, H. H.; Petersen, G. Chem. Phys. Lipids 2000, 108, 135.

(3) Ray, B. L.; Painter, G.; Raetz, C. R. H. J. Biol. Chem. 1984, $259,4852$.

(4) Devadas, B.; Lu, T.; Katoh, A.; Kishore, N. S.; Wade, A. C.; Mehta, P. P.; Rudnick, D. A.; Bryant, M. L.; Adams, S. P.; Li, Q.; Gokel, G. W.; Gordon, J. I. J. Biol. Chem. 1992, 261, 7224.

(5) Furutani, T.; Ooshima, H.; Kato, J. Enzyme Microb. Technol. 1997, 20, 214

(6) Furutani, T.; Ooshima, H.; Kato, J. Enzyme Microb. Technol. 1996, 19, 578.

(7) Fernandez-Perez, M.; Otero, C. Enzyme Microb. Technol. 2001, $28,527$.

(8) Hildreth, J. International Patent Application No. WO 83 04412, 1983.

(9) Maugard, T.; Remaud-Simeon, M.; Petre, D.; Monsan, P. Tetrahedron 1997, 53, 5185.

(10) Hildreth, J. Biochem. J. 1982, 207, 363.

(11) Sarney, D. B.; Vulfson, E. N. Tibtech 1995, 13, 164

(12) Infante, M.; Molinero, J.; Bosch, P.; Julia, M. R.; Erra, P. J. Am. Oil Chem. Soc. 1989, 66, 1835.

(13) Mhaskar, S. Y.; Lakshminaratana, G. J. Am. Oil Chem. Soc. 1992, 69, 643.

(14) Bilbo, R. E.; Prislinger, A. Alkanolamides. Soap/Cosmet./Chem. Spec. 1989, 44.

(15) Melzer, V.; Vollhardt, D. Phys. Rev. Lett. 1996, 76, 3770.

(16) Vollhardt, D.; Melzer, V. J. Phys. Chem. B 1997, 101, 3370.

(17) Melzer, V.; Weidemann, G.; Vollhardt, D.; Brezesinski, G.; Wagner, R.; Struth, B.; Möhwald, H. J. Phys. Chem. B 1997, 101, 4752.

(18) Melzer, V.; Weidemann, G.; Vollhardt, D.; Brezesinski, G.; Wagner, R.; Struth, B.; Möhwald, H. Supramol. Sci. 1997, 4, 391.

(19) Melzer, V.; Vollhardt, D. Prog. Colloid Polym. Sci. 1997, $105,130$.

(20) Melzer, V.; Vollhardt, D.; Brezesinski, G.; Möhwald, H. J. Phys. Chem. B 1998, 102, 591.

(21) Melzer, V.; Vollhardt, D.; Weidemann, G.; Brezesinski, G.; Wagner, R.; Möhwald, H. Phys. Rev. E 1998, 57, 901.

(22) Vollhardt, D.; Melzer, V.; Fainermann, V. B. Thin Solid Films 1998, 327-329, 842 .

(23) Melzer, V.; Vollhardt, D.; Brezesinski, G.; Möhwald, H. Thin Solid Films 1998, 327-329, 857.

(24) Melzer, V.; Weidemann, G.; Wagner, R.; Vollhardt, D.; DeWolf, C.; Brezesinski, G.; Möhwald, H. Chem. Eng. Technol. 1998, 21, 44.

(25) Fainerman, V. B.; Vollhardt, D. J. Phys. Chem. B 2003, 107, 3098.

(26) Vollhardt, D.; Wagner, R. J. Phys. Chem. B 2006, 110, 14881.

(27) Henon, S.; Meunier, J. Rev. Sci. Instrum. 1991, 62, 936.

(28) Hönig, D.; Möbius, D. J. Phys. Chem. 1991, 95, 4590.

(29) Vollhardt, D. Adv. Colloid Interface Sci. 1996, 64, 143.

(30) Mendelsohn, R.; Brauner, J. W.; Gericke, A. Annu. Rev. Phys. Chem. 1995, 46, 305 .

(31) Flach, C. R.; Gericke, A.; Mendelsohn, R. J. Phys. Chem. B 1997, 101, 58.

(32) Andreeva, T. D.; Petrov, J. G.; Brezesinski, G.; Möhwald, H. Langmuir 2008, 24, 8001-8007.

(33) Lepère, M.; Muenter, A. H.; Chevallard, C.; Guenoun, P.; Brezesinski, G. Colloids Surf., A 2007, 303, 73.

(34) Als-Nielsen, J.; Jacquemain, D.; Kjaer, K.; Leveiller, F.; Lahav, M.; Leiserowitz, L. Phys. Rep. 1994, 246, 251.

(35) Kjaer, K. Physica B 1994, 198, 100.

(36) Kaganer, V. M.; Möhwald, H.; Dutta, P. Rev. Mod. Phys. 1999, $71,779$.

(37) Lepere, M.; Chevallard, C.; Brezesinski, G.; Goldmann, M.; Guenoun, P. Angew. Chem., Int. Ed. 2009, 48, 5005.
(38) Vollhardt, D. Adv. Colloid Interface Sci. 1996, 64, 143.

(39) Fradin, C.; Daillant, J.; Braslau, A.; Luzet, D.; Alba, M.; Goldmann, M. Eur. Phys. J. B 1998, 1, 57.

(40) Kaganer, V. M.; Brezesinski, G.; Möhwald, H.; Howes, P. B.; Kjaer, K. Phys. Rev. E 1999, 59, 2141.

(41) Vollhardt, D.; Brezesinski, G.; Siegel, S.; Emrich, G. J. Phys. Chem. B 2001, 105, 12061.

(42) Brezesinski, G.; Vollhardt, D. ChemPhysChem 2008, 9, 1670.

(43) Brezesinski, G.; Vollhardt, D.; Iimura, K.; Cölfen, H. J. Phys. Chem. C 2008, 112, 15777. 